\title{
Staged interventional and surgical treatment of tetralogy of Fallot with critical stenosis of proximal aortic arch in premature hypotrophic newborn
}

\author{
Ireneusz Haponiuk ${ }^{1}$, Maciej Chojnicki², Radosław Jaworski², Aneta Szofer-Sendrowska², Konrad Paczkowski², \\ Katarzyna Gierat-Haponiuk ${ }^{3}$
}

${ }^{1}$ Chair of Physiotherapy, Department of Pediatric Cardiac Surgery, Mikolaj Kopernik Hospital, Gdansk, Poland

${ }^{2}$ Department of Pediatric Cardiac Surgery, Mikolaj Kopernik Hospital, Gdansk, Poland

${ }^{3}$ Department of Rehabilitation, Medical University of Gdansk, Gdansk, Poland

Adv Interv Cardiol 2016; 12, 1 (43): 65-67

DOI: $10.5114 /$ pwki.2016.56953

\section{Introduction}

Tetralogy of Fallot (ToF) is the most common cyanotic congenital heart defect diagnosed in newborns. Depending on the degree of right ventricle outflow tract stenosis and clinical signs of cyanosis, the pathology necessitates intensive medical, interventional and surgical treatment in early infancy with anatomic correction before the first year of life. ToF is often accompanied by additional cardiovascular defects and congenital abnormalities apart from the circulatory system. Complex stenosis of the right ventricle outflow tract (RVOT) in ToF is rarely accompanied by any stenosis of the left ventricle outflow structures [1]. Regular treatment becomes more problematic in borderline low body weight patients suffering from prematurity and hypotrophy (birth weight $<2.5 \mathrm{~kg}$ ), who usually do not meet regular criteria for surgery and cardiac interventions.

\section{Case report}

A 2-month-old premature hypotrophic newborn boy, $2.3 \mathrm{~kg}$ b.w., was referred to the emergency department with severe cyanosis in the course of postnatally diagnosed ToF. Peripheral saturations on air were less than $70 \%$. Apart from dysmorphia features, the boy underwent resection of an additional thumb. Also he had a history of intensive treatment of congenital pneumonia in a different institution. Despite the cyanosis there was an evident pressure gradient of upper-to-lower limbs of $40 \mathrm{~mm} \mathrm{Hg}$, with diminished pulse in the femoral artery. Initial echo showed typical morphology of ToF with right aortic arch, malalignment $12 \mathrm{~mm}$ ventricular sep- tal defect (VSD), non-restricted right-to-left shunt, 70\% overriding aorta, hypertrophied right ventricle (RV) and a severely stenotic, dysmorphic pulmonary valve (PV) in the hypoplastic pulmonary trunk (PA). Peripheral pulmonary arteries had acceptable size with McGoon index $>1.5$, although intracardiac defects were accompanied by critical stenosis in the proximal aortic arch of $2 \mathrm{~mm}$ width and 40-50 $\mathrm{mm} \mathrm{Hg}$ echocardiographic pressure gradient. During cardiac catheterization there was revealed right aortic arch, internal carotid arteries right and left extending from the ascending aorta, and the right subclavian artery from the distal part of the aortic arch. The left subclavian artery was filling up to the collateral circulation. Probably the left subclavian artery arose from the closed ductus arteriosus. The patient was qualified for cardiology intervention with balloon plasty of the aortic arch (Figure 1). During the procedure an early restenosis was found, because of high stiffness of the stenotic area, so we decided to implant a stent. Direct measurements of the stenotic aorta precluded the use of a regular pediatric aortic stent in the premature arch; therefore a coronary stent was implanted (Multi-Link $84 \mathrm{~mm} / 8 \mathrm{~mm}$, Abbott Vascular, Illinois, USA), with its deployment to $4.5 \mathrm{~mm}$ (Figure 2). Control transthoracic echocardiography (TTE) showed unrestricted flow in the aortic arch. The boy was then referred for percutaneous RVOT balloon plasty 7 days later. After right ventriculography that showed a severely stenosed PV and $5 \mathrm{~mm}$ width RVOT, a single approach triple balloon plasty was performed. The direction of flow over the unrestricted VSD changed for left-to-right. Because of previously diagnosed right-sided iliac and femoral artery thrombosis

\section{Corresponding author:}

Ireneusz Haponiuk, Department of Pediatric Cardiac Surgery, Mikolaj Kopernik Hospital, 1-6 Nowe Ogrody St, 80-803 Gdansk, Poland, phone: +48 5876404 90, e-mail: ireneusz_haponiuk@poczta.onet.pl

Received: 2.05.2015, accepted: 28.06.2015. 


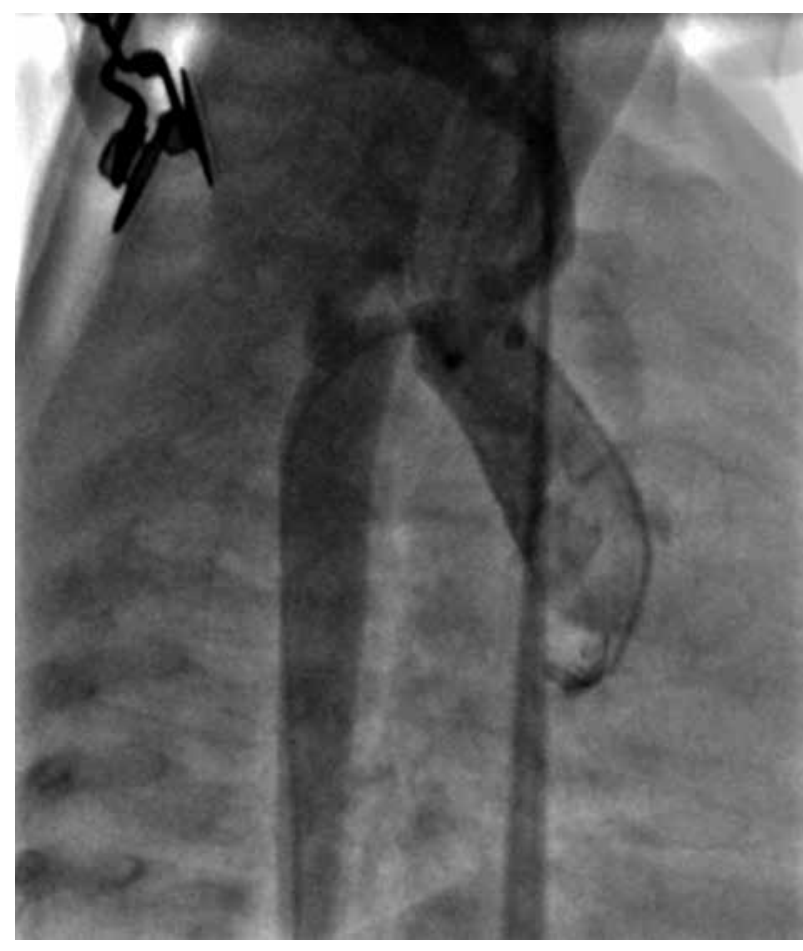

Figure 1. Native stenosis in proximal right aortic arch in child with tetralogy of Fallot $-2.3 \mathrm{~kg}$ b.w. premature newborn

local fibrinolysis was performed (120 min alteplase infusion). During administration of intravenous alteplase $(0.2 \mathrm{mg} / \mathrm{kg} / \mathrm{h})$ with heparin $(15 \mathrm{U} / \mathrm{kg} / \mathrm{h})$ the arterial flow was restored in the thrombotic femoral vessels. Oral aspirin $3 \mathrm{mg} / \mathrm{kg}$ b.w. was administered. During a month of treatment in the Department of Pediatrics the symptoms of dysphagia with anxiety and cyanosis, with bidirectional interventricular shunt features in control TTE, were observed. Regular modified Blalock-Taussig shunt (BT) was performed in the $2.8 \mathrm{~kg}$ boy via right-sided thoracotomy with a $3.5 \mathrm{~mm}$ Gore-Tex tube. After the shunt the baby was referred for additional RVOT balloon plasty. At the very beginning of the anesthesia induction, despite adequate shunt flow, he fell into a severe cyanotic spell with the need of intensive medical treatment and cardiac massage. The procedure was abandoned. Three days later the $2.9 \mathrm{~kg}$ b.w. premature patient underwent a successful surgical correction of the ToF with extensive resection of the hypertrophied $\mathrm{RV}$ and an intraoperative hybrid pulmonary balloon plasty. The stent was removed with a resection of stenosed aortic segment and combined end-to-end anastomosis between the brachiocephalic trunk and the left carotid artery was done with means of deep hypothermia circulatory arrest (DHCA). The patient was extubated on the operative day. Transient atrioventricular (AV) block with temporal epicardial pacing was observed in the early postoperative course. Further treatment was uneventful. Because of oral feed-

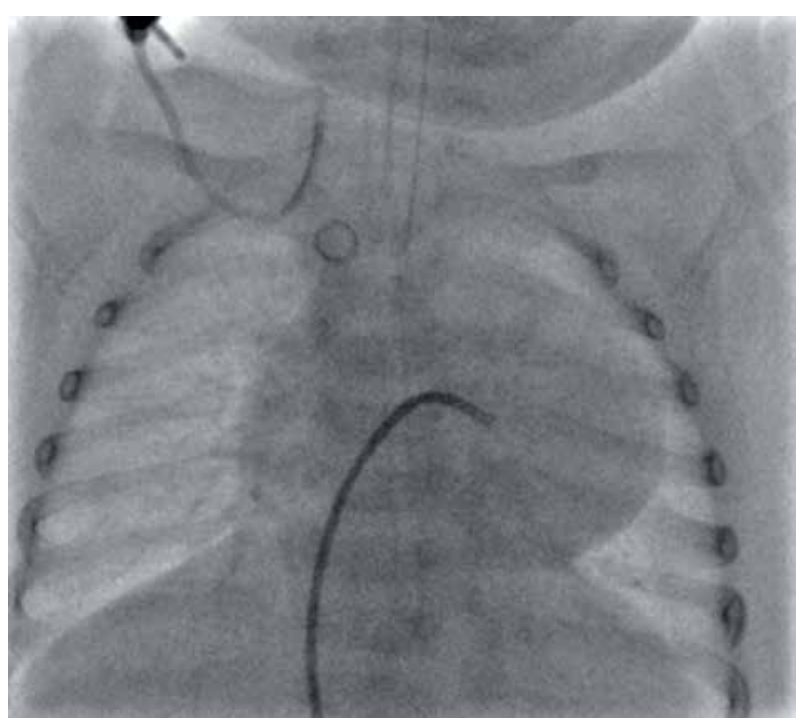

Figure 2. Coronary stent directly implanted into the area of proximal arch native stenosis (Abbott $4 \mathrm{~mm} / 8 \mathrm{~mm})-2.3 \mathrm{~kg}$ b.w. premature newborn

ing difficulties the boy was treated in the Department of Gastroenterology and then discharged home 3 weeks after surgery. He was regularly followed up in the outpatient clinic. His echocardiography showed closed VSD, mild residual stenosis of RVOT up to $35 \mathrm{~mm} \mathrm{Hg}$ and free flow in the aortic arch. The patient returned to his regular rehabilitation protocol, typical for resolving physical and developmental problems of prematurity.

\section{Discussion}

Our clinical routine is an early correction of ToF with staged balloon plasty of RVOT, $\beta$-blocker treatment in patients with evident RVOT hypertrophy and preventive BT shunt in any signs of emergency because of severe hypoxia [2]. We prefer a comprehensive therapy from the very birth, with prenatal diagnostics and precise plans of treatment. In our observation the strategy with staged balloon RVOT plastics, with RVOT stenting in some cases, reduces muscular hypertrophy of the right ventricle, and improves the early postoperative course after the correction of ToF.

Stenosis of the proximal aortic arch is rarely observed in patients with ToF. The aortic stenosis causes additional systemic overload, with concomitant typical signs of cyanosis. The situation could be difficult in the initial diagnostics; thus we stress precise echocardiography of every problematic patient. The procedure of stenting the arch resolved the problem with left ventricle overload before anatomic correction of ToF.

Our strategy is applied in every patient; therefore we consciously suppose that staged interventional treatment could be beneficial also for borderline patients with accompanying cardiovascular anomalies. We proved that 
low body weight and prematurity are independent surgical risk factors for babies with complex cardiac problems [3].

Following our experience, low body weight is no longer a contraindication for percutaneous interventional procedures. We successfully introduced invasive treatment even in premature babies. Nevertheless, we keep in mind that cardiac interventions in small premature babies are related to additional problems, which cause the need of additional treatment [4].

In the present case, an adequate shunt flow did not protect the baby from cyanotic spells, which could be related to additional comorbidities (prematurity, low gestational age, bronchopulmonary dysplasia, low body weight) [5].

Effective treatment of congenital cardiovascular problems in the early infancy of the present premature patient, despite additional clinical disadvantages, supported his intensive general rehabilitation [6].

\section{Conclusions}

Modern alternative hybrid and staged interventional strategies supported with intraoperative imaging combine the advantages of surgery and interventional cardiology to treat more complicated patients despite their suboptimal condition.

\section{Conflict of interest}

The authors declare no conflict of interest.

\section{References}

1. Kenny D, Hijazi ZM. Coarctation of the aorta: from fetal life to adulthood. Cardiology J 2011; 16: 487-95.

2. Santoro G, Bigazzi MC, Palladino MT, et al. Transcatheter palliation of tetralogy of Fallot with pulmonary artery discontinuity. Tex Heart Institute J 2005; 32: 102-4.

3. Kansy A, Tobota Z, Maruszewski P, Maruszewski B. Analysis of 14843 neonatal congenital heart surgical procedures in the European Association for Cardiothoracic Surgery Congenital Database. Ann Thorac Surg 2010; 89: 1255-9.

4. Kretschmar O, Dähnert I, Berger F, et al. Interventional treatment of congenital heart defects in infants with a body weight up to 2,500 grams. Z Kardiol 2000; 89: 1126-32.

5. Juściński J, Haponiuk I, Chojnicki M, et al. Operative treatment of tetralogy of Fallot with concomitant correction of anomalous origin of the left pulmonary artery from Kommerell's diverticulum. Kardiochir Torakochir Pol 2014; 11: 336-8.

6. Wojtalik M. Tetralogy of Fallot. Physical work capacity after its total correction. Kardiol Pol 1992; 36: 215-9. 